\title{
A New Approach Teaching Mathematics, Modelling and Simulation
}

\author{
Stefanie Winkler Andreas Körner Felix Breitenecker \\ Institute for Analysis and Scientific Computing, Vienna University of Technology, 1040 Vienna, Austria \\ \{stefanie.winkler, andreas.koerner, felix.breitenecker\}@tuwien.ac.at
}

\begin{abstract}
This paper introduces two different e-Learning environments. Both are used at the Vienna University of Technology to support the courses and exercises in mathematics. There are different level of courses. On the one hand there is a refresher course to support new students who might had some time off before starting their study as well as flatten different school levels of mathematics. On the other hand there are regular mathematical courses in the first two to three semester. Due to improved and advanced possibilities offered by the environment in the last years the system enables the integration of simulation examples. In 2006 the research group Mathematical Modelling and Simulation (MMS) developed an individual web-server to provide students with simulation examples. This server was used in the lectures as well as for practice at home. In the last year also a combination of Moodle and this web application was used to perform tests. This paper should give a short introduction in both systems and compare their advantages and disadvantages. In the outlook a new possibility is presented to combine the advantages of both presented systems.
\end{abstract}

Keywords: modelling and simulation, education, blended learning, case studies

\section{Introduction}

In 2004 the research group started to use online tools to improve the quality or at least the administration part of lectures. The first step was using a moodle based platform. The moodle installation was made by an group at the university and is still supervised by the same group. They are as well developing various additional plugins for the moodle based university website. The website is mainly used to organize lecture notes and additional materials. There is also another web application used for lectures dealing with modelling and simulation. This environment has been developed in 2006 and is called Mathematical, Modelling and Tools (MMT). In general it is a simple content management system but with the possibility to connect to simulation environments. This eLearning system is explained in Section 2. (Winkler et al., 2010)

Due to the fact that the research group Mathematical Modelling and Simulation (MMS) was one of the first users of the moodle based university platform, developed the MMT server and won an E-Learning award in 2007 the vice rector for academic affairs commissioned the research group to organize and administrate a refresher course using b-learning. This refresher course was initialised to help students starting their studies subsequently after school. The content of this course is a summary of mathematical knowledge students should have gained at school. The structure and the used eLearning system is explained in Section 2.

\section{MMT - Mathematics, Modelling and Tools}

\subsection{Structure of MMT}

The requirements for the website include the possibility to use modelling and simulation examples online. The students should be able to execute a certain simulation example and receive the generated output plot. Another feature is experimenting with these simulations in some kind. The realization enables students to change different parameters of the system and generate the new output to get an idea of the influence these parameters have.

In the last years there where several improvements in the environment. At the beginning the usage of simulation software was restriced to Matlab alone. The current examples show a wider field. There are examples running in Matlab, Simulink, Octave, Anylogic as well as Java. The expansion including Simulink lead to one problem. Compared to all other examples implementations in Simulink need two different $\mathrm{m}$-files to work properly. In case of Matlab and Octave it is enough to simply execute one program file. The Analogic examples use so called Java Applets which represent the interface between Anylogic and webserver.(Körner et al., 2011) In Figure 1 and Figure 2 the structure of the MMT Server and the examples available is depicted. As you can see in Figure 1 on the left hand side all examples are assigned to different topics. In one topic there are more than one implementation. For example different cases of the pendulum model are available. All the pendulum examples are in the folder "'Pendulum"', as shown in Figure 1 marked with number 1. Therefore the theoretical and mathematical basics are explained on the first site of the main topic. The different model implementations explain only the current used method and the different parameters, as marked with num- 


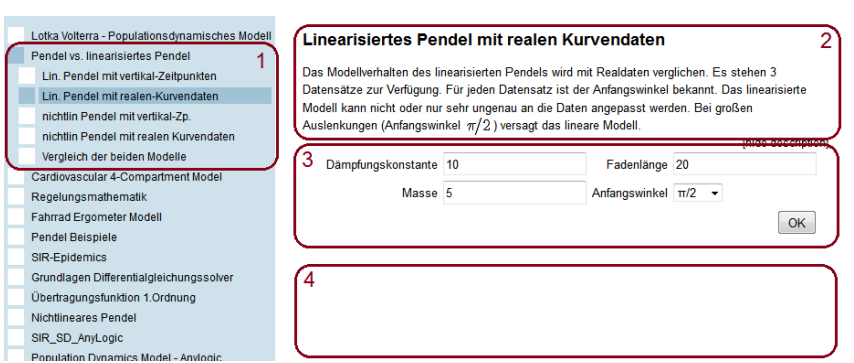

Figure 1. Structure of the MMT system.

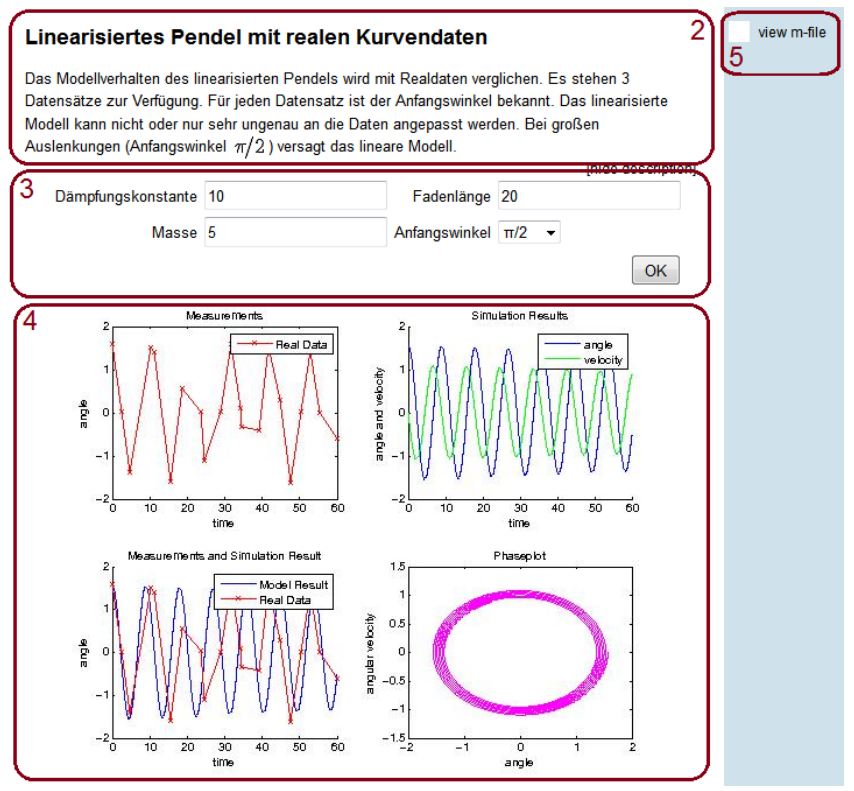

Figure 2. Structure of MMT examples.

ber 2 in Figure 1. In order to know which parameter influences the model output how the students have the possibility to change one or in most examples more than one parameter values. Section 3 shows the parameter area. After setting parameter the student has to summit the parameters by clicking $O K$ which leads to the execution of the model. If the example is more complicated it might take some time but usual after some seconds the output plot appears as shown in Figure 1 and 2. Section 5 of Figure 2 offers a link to an $\mathrm{m}$-file. In general this section enables lecturer to upload additional material for the different examples as well. For sure there will be the file containing the corresponding algorithm. It does not matter which simulation environment was used for the implementation. Students have the possibility to download the file and execute or adapt it on their own computer to see how such examples are structured and which commands are used to implement for example the pendulum. (Bicher et al., 2013)

\subsection{Usage of MMT}

At the moment there are nearly 700 examples stored on the web server. The examples cover various fields of application. Some of the topics are even implemented in different environments to show various realizations or even ap- proaches simulating continuous and discrete models. The examples are used for different courses. The modelling and simulation courses are offered to students of electrical engineering, information technology, mechanical engineering as well as mathematicians. Due to the fact that the knowledge of these groups are not identically the courses are organized differently. Every course contains a certain selection of topics to satisfy the needs of students from different field of studies.

In most of the lectures it is also necessary to pass a test. Up to now these tests are implemented in Moodle. In general the test consists of theoretical and practical questions. The theoretical questions deal with different approaches or even mathematical basic questions. Regarding the practical part the experiments on the MMT server are slightly modified to allow such test questions. In some examples the students have to adapt the parameters of the model to gain certain information of the model behavior, e.g. the state point of a model. Then the results of the practical examples have to be inserted into the Moodle question. Due to the complex structure no randomization in the examples is possible.

\section{Maple T.A. - Maple Testing and As- sessment}

In 2008 the research group was commissioned to initialize a refresher course. There were different requirements the course had to fulfill. The course should be held in the first two weeks of the semester and enables high participation. Additionally the course structure should provide some kind of E-learning support. Due to the fact that the topics of this course are just mathematical subjects the MMT server was not providing a suitable structure. A system called Maple T.A. was chosen.(Urbonaite and Winkler, 2013)

\subsection{Structure MTA}

Maple T.A. is an interface developed by Maplesoft. In 2008 the software was quite new. It provides an interface to create mathematical questions as well as theoretical questions. The main aspect of the system is the possibility to create practice and test assignments. Therefore it is not only a content management system where different questions are stored but an environment where students can exercise and improve their calculation skills. The administration of examples as well as users is quite easy. In order to create a new question one has to decide which type of question is required. There are different types available, e.g. Multiple Choice, Numeric, Algebraic, graphical questions and so on. For the algorithm the ordinary maple commands are available. The initialization of variables is different but despite of that a user of Maple won't have any problems creating a basic mathematical questions. To give an example a regular if-command in Maple looks like

$$
\text { if } a>b \text { then } a \text { else } b \text { end if . }
$$


If one uses Maple T.A. syntax to get the same result it can be written like

$$
\text { if }(g t(a, b), a, b)
$$

For using Maple T.A. to create examples, it is not necessary to know commands of Maple T.A. On the other hand it is helpful to know some of the short devices because they are develop to avoid constant use of the underlying maple kernel, leading to lower capacity requirements. In some cases it is better to use special commands of Maple T.A. Below a short code of a very easy example is given to show the difference to Maple.

$$
\begin{aligned}
& \$ a=\operatorname{range}(1,5) ; \\
& \$ b=\operatorname{range}(1,6) ; \\
& \$ c=\operatorname{range}(1,3) ; \\
& \$ a n s=\text { maple ("if } \$ a>\$ b \text { and } \$ a>\$ c \text { then } \$ a \text { elif } \\
& \quad \$ b>\$ a \text { and } \$ b>\$ c \text { then } \$ b \text { else } \$ c \text { end if"); }
\end{aligned}
$$

All the created questions can then be combined to assignments associated to a certain topic of the lecture. The assignments can be used during exercises as example resource and of course students can practice examples anytime at home. One advantage of the system is an easy administration of examples and course assignments. Another, even more important benefit, is the usage of a computer algebra system regarding grading of examples. The grading can be easily formalized in a mathematical algorithm ignoring equivalent transformations. On the other hand the question task can be randomized using different parameters in the formulation of even randomize functions, e.g. for calculating a derivative. The variety of the examples increases and students can practice the same assignment more often without repeating exactly the same examples.

\subsection{Usage MTA}

In the beginning the questions used in the refresher course were very simple. In 2010 the usage of the system was extended. Not only the refresher course but also the basic mathematical courses in the first and second semester were using the system were intense. The level and complexity of the questions increased. This lead to another problem. The grading algorithms had to be improved and get more complex as well. In order to support the acceptance of the system among students the grading provided the possibility to gain partial points if the result is partly right. Two different libraries were established to enable teachers to give partial points if a result is more complicated. On the oterhe hand the library also simplifies the process of creating examples. In Figure 3 an example using the partial grading is shown. This example also uses the second library containing commands to generate randomized matrices with defined properties. The libraries are as well used to guarantee a certain structure of the solution in order to assure similar levels despite randomized factors. The examples cover all topics of the mathematical

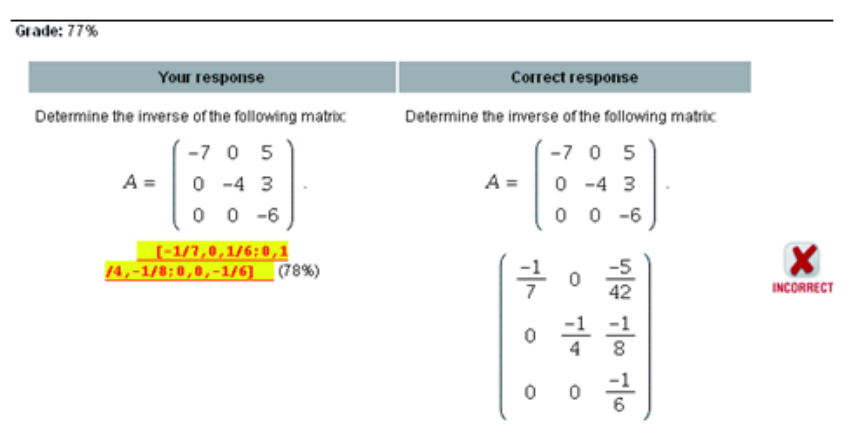

Figure 3. An example working with partial grading.

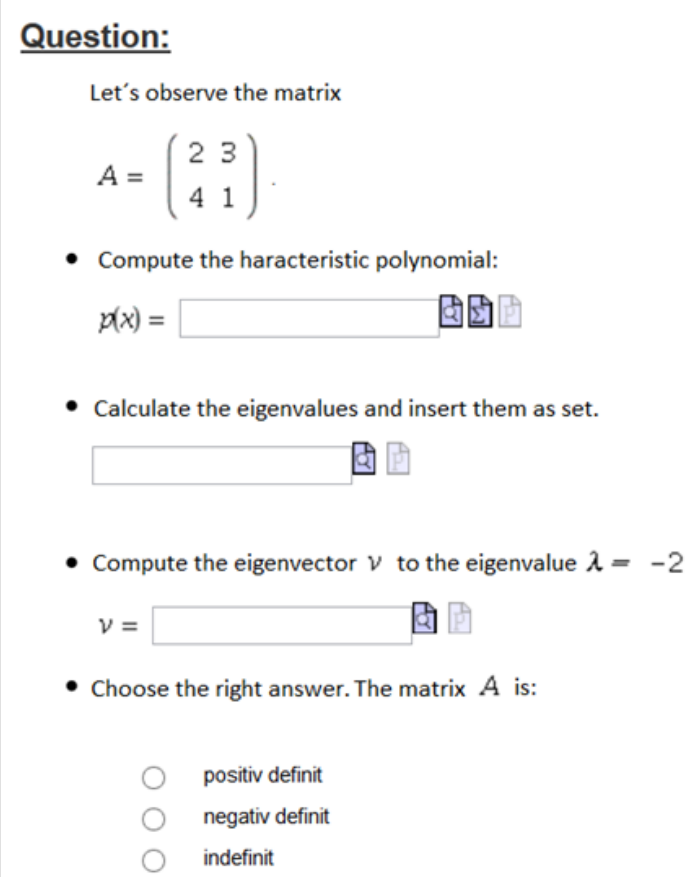

Figure 4. An example used in the written exam.

courses for students of electrical engineering. Examples dealing with Analysis in one and two dimensions, Linear Algebra as well as Vector Analysis and complex analysis are available. Figure 4 shows an example of the chapter Linear Algebra which is also using the libraries mentioned above. Since some years the system is also used to realize exams. Before then the system was used to support the exercises and perform midterm tests. Students have to pass 2 of the 4 provided tests each semester in order to pass the exercise part. For the lecture they have to pass a separate exam. Compared to the small tests during the semester, which only take 30 minutes, the exam lasts 2 hours. This exam is a written test and afterwards a short oral exam follows. Since 2012 the students can decide if they want to do the test as an ordinary written exam or on the computer system. Figure 4 shows a possible question for the exam. The examples are the same but in the online example the variables and functions are changing. Therefore it 
The graph below shows the fucntion $d^{x}$ with the changing variable $a$. $a=\left[\begin{array}{lllllllllllll}4 & 1 & 1 & 1 & 1 & & 1 & 1 & 1 & 1 & 1 & 1 \\ 0 & 1 & 2 & 3 & 4 & 5 & 6 & 7 & 8 & 9 & 10\end{array}\right.$

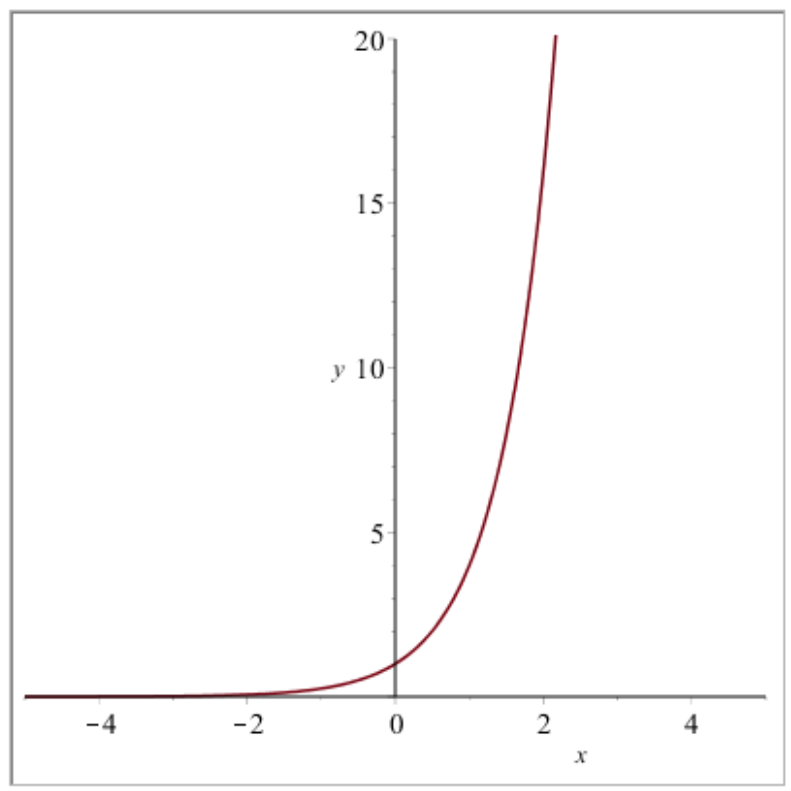

Figure 5. A simple mathapp question used to explain the basics of exponential functions.

is nearly unable to cheat at the examination.

\subsection{Mathapps}

Due to the fact that the system evolved over the past years there are new interesting features. As mentioned before the development of a test in the web sever MMT is not so easy. A disadvantage is the lack of flexibility regarding randomized test questions. As explained above Maple T.A. enables a wide range of possibilities to randomize parameters and functions of questions. So it would be very feasible to use Maple T.A. for the modelling and simulation courses.

In order to establish simulation examples in Maple T.A. an explanation of Mathapps is necessary. Since some years Maplesoft enables the creation of Mathapps. In general it is a certain form of Maple Worksheets. Before this invention lectures used as well Maple Worksheet to explain mathematical basics benefiting from the algebraic properties of Maple. Compared to an ordinary worksheet Mathapps additionally enable integration of sliders, figures and controllers to design an interactive area as shown in Figure 5. After that an exponential function with parameter $a$ is given. After setting the parameter $a$ using the slider, the graphic is loaded automatically. Such mathapps can be used to explain mathematical principles more easily using the figure of the problem. Regarding modelling and simulation this improvements enables the creation of example similar to MMT examples from above. Students have the possibility to change the parameter and after sub- mitting the new parameter output and figure is actualized automatically.

The implementation using Mathapps can be divided into three different parts. On the one hand there is the so called Startup Code which is executed during loading the question and generates the output plot. In Section 2 it is described that in the beginning there is no output on the MMT server. Students can only see the text describing the example and the parameter area. After submitting the parameter the output appears. In Maple T.A. this can be done automatically. In order to create a efficient example the used algorithm in the Startup Code defines a procedure and therefore can be used again.

If the students submits the parameters using the button start the procedure defined in the Startup Code is used to update the output plot. The last part is the grading. There are two different options. On the one hand it is possible to write a grading procedure as well inside the Startup Code. This grading routine then can be used directly in Maple T.A. Another possibility is to formulate the algorithm in Maple T.A. itself. It does not matter which option is chosen. In both cases the current values of all sliders can be used to determine if the chosen input variables students chose are right or wrong.

\subsection{MTA - Moodle Connector}

In all mentioned cases there is a Moodle course where the lecture notes and materials are organized. A student who enrolls at Vienna University of Technology receives a unique registration number and a password for the university system for course administration. With this account the student can enter the course administration website as well as the moodle based E-Learning website. In previous times the student had to enter the number and password of the university to get an account for Maple T.A. So a student has to go through 3 different websites to get to Maple T.A. Since 2013 it is possible to integrate the Maple T.A. into the e-learning platform of the Vienna University of Technology. This connection is a data interface. If a student registers for the course on the e-learning platform from the university the student is able to get to the examples directly through a link on the course page as shown in Figure 6. There is no need for an additional account in Maple T.A. All results the students gain in the different courses are recorded in Maple T.A. and sent to moodle. This is very useful for administration because also the grade in the end of the semester is created in the moodle system. Therefore the test results can be involved in a formula to generate the over all grade.

\section{Case Study}

In Section 3.3 the basics of Mathapps created in Maple are explained. Using this tool it is possible to create examples similar to the examples in MMT as shown in Section 2. An implementation of a pendulum example in MMT is shown in Figure 7. Analog Figure 8 presents the realization of the same example inside Maple T.A. using a Mathapp. 
3. Übung: 11. - 13.11.2013

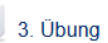

3. Übung

Unter den folgenen Links finden Sie Übrungsbeispiele zu den Themen des 3.Übı unter anderem Integrale über Flächen und Körper.

Flächenintegrale

$\checkmark$ Körperintegrale

Q Beispiele zu Satz von Gauß und Stokes

1.Klausur, 20.11.2013

Unter dem folgenden Link finden Sie die erste Klausur aus den VU Mathematik 3 Modus: Es dürfen nur Taschenrechner verwendet werden, die nicht programmie "einzeilig") sind. Bringen Sie eigenes Papier und Schreibutensilien für Nebenrect nicht herangezogen. Unterlagen sind keine erlaubt!

\section{(8. 1.UE-Test}

Figure 6. Usage of the moodle Connector to link Maple T.A. assignments.

\section{Mathematisches Pendel}

Wir betrachten zunächst das klassische mathematische Pendel das der Gleichung $m l \dot{\varphi}(t)=-m g \sin (\varphi(t))-l d \dot{\varphi}(t)$

genügt

Man betrachtet dieses Beispiel durch Parametervariation der Länge $l$ des masselosen Fadens, des Startwinkels $\varphi(0) \in(-\pi / 2, \pi / 2)$, der Startgeschwindigkeit $\dot{\varphi}(0)$ und der Dämpfungskonstanten $d$
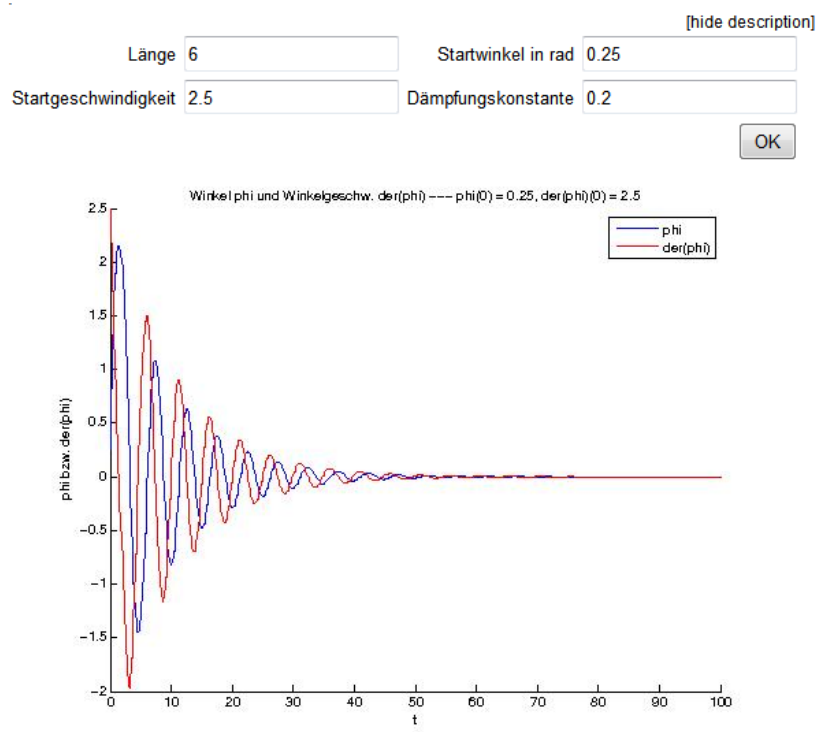

Figure 7. A pendulum example on MMT server.

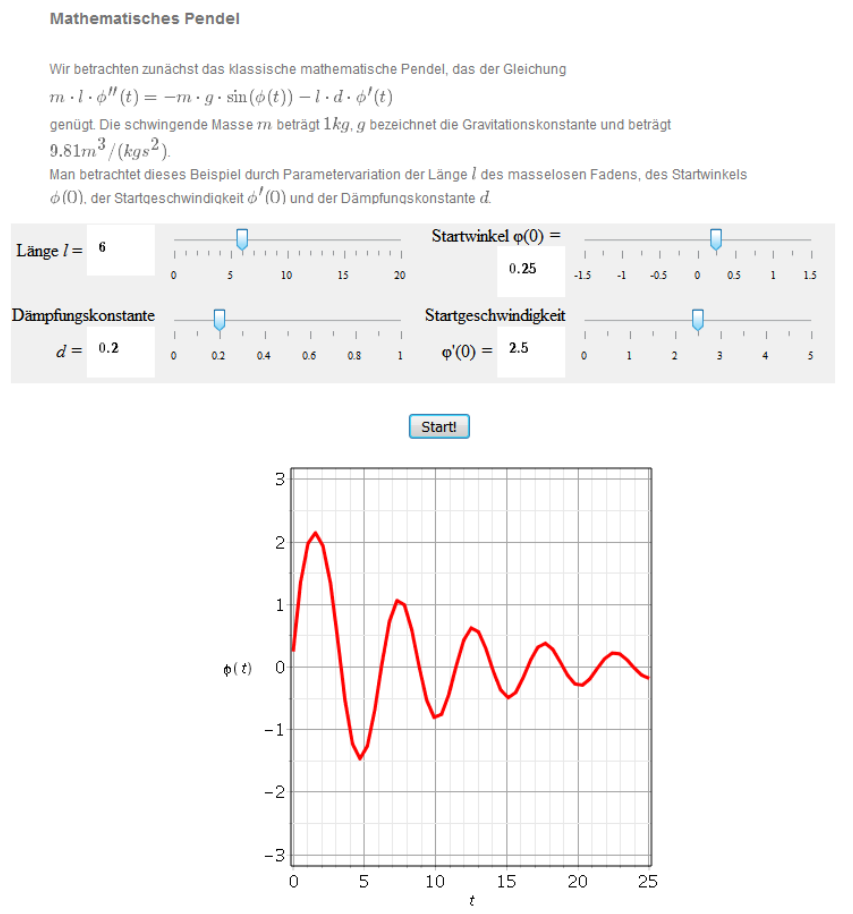

Figure 8. Implementation of a pendulum example in Maple T.A. with Mathapps.

The basic differential equation used to describe the behavior of a pendulum are simple. The implementation of this differential equations is easier done in Matlab or any other numerical environment. It is a greater challenge to implement the same behavior in Maple. In the last years also Maple made some improvements to enable the numerical calculations which are necessary for such Mathapps. Ignoring the fact that the implementation might be easier in Matlab the qualitative behavior of the model is recognizable in both realizations. Both environment allow a default setting by the model. The student can then start experimenting using the sliders to change the parameters or simply insert the parameter value. An advantage of the slider is a restriction of the parameter range. Also in MMT the range of all parameters is restricted but the student would get an error message that a certain parameter value is not possible and then change the parameter in order to execute the example again with a possible value. In case of the Mathapp this can not happen because the student can see the range of the slider. Comparing the two figures the output plot in Maple might be not used to its full potential. Further improvements are possible to enable an easy understanding.

The implementation might be easier using Matlab. The example in Maple T.A. might seem clearer for the students. The area where the parameters can be changed is neatly arranged in the Mathapp implementation. Even the symbols used for the parameter change, if it is a slider a controller or something completely different, can be adjusted to the meaning of the parameter and its variation.

As mentioned in Section 2 there is a test realized using 
moodle and MMT in combination. Regarding the lack of randomization in this test as well as level of complexity of questions Mathapps offer more possibilities. One the one hand for grading Mathapps in Maple T.A. every command and library of Maple can be used. Therefore the possible issues which can be covered with the same modelling and simulation question are very variable and different from MMT. Another aspect is the very feasible administration of tests. The acronym T.A. stands for Testing and Assessment therefore the interface provides all setting such tests could need. On the MMT server it was necessary to create a separate question for the test. In Maple T.A. it is possible to use the same Mathapp for practicing and testing. But it is also possible to use the examples in the lecture to explain the basic ideas of the model and the influences of different parameters.

Up to now only some of the modelling and simulation examples are transferred to Maple T.A. Of course it is very easy to formulate all the theoretical qeustions in Maple T.A. For the simulation examples it takes some time to figure out how to implement differential equations efficient in Maple. It is not possible to use the algorithm used in Matlab because the general idea of the two software are completely different.

Despite all the new possibilities of Maple T.A. there are also disadvantages. It is not possible to transfer all the eamples to Maple T.A. because some of the examples are based on Anylogic, Simulink and Java. These Tools are not supported by Maple so we can not use these examples anymore.

\section{Conclusions}

The comparison of both environment shows that there is no such thing as the best choice. Both systems have their advantages and disadvantages. MMT enables examples using different software products combining them in one content management system. The output on the MMT system shows more detail and potential. But the problem might be that there are much more possibilities in Maple than used in Figure 8. The Mathapps and outputs should be implemented more carefully and then it might be possible to generate outputs as detailed as in Matlab. In Maple T.A. it is not possible to add additional materials. Instead it would be possible to but additional material into the moodle course.

Maple T.A. offers some nice features regarding testing. It is very easy to provide examples in a feasible way for testing and assessing students. Additionally the usage of the moodle Connector mentioned above enables students to connect to the system without even knowing that they changed the platform. At the MMT server every course has its own authorization code. In Maple T.A. practicing and testing is now personalized. The appearance of the examples using sliders and controllers improved. The main advantage of the system might be again the great variety using random variables and function. And as a con- sequence also the grading offers much more possibilities.

\section{Outlook}

The conclusion shows that even Maple T.A. which is developed for testing and assessment misses some features. Especially regarding using a combination of different tools. There is an additional environment developed by Maplesoft which could help to erase remaining problems regarding modelling and simulation examples. An aspect which would be nice to extend are teaching possibilities. For Testing Maple T.A. has very useable features. In order to enable more teaching aspects using Maple T.A. Maplesoft came up as well with a solution. The new software is call Möbius (Maplesoft, 2016) and combines teaching and testing aspects. It is a combination of content management system and Maple T.A. but in a more interactive way. It is possible to design something similar to a textbook site including Mathapps as well as Maple T.A. questions.

Imagine a text explaining perhaps the basics of exponential functions. Next an interactive action, e.g. Figure 5 , is included. Then there are some examples with its solution step by step. Afterwards an example from Maple T.A. can be integrated. This example actualizes every time. Students can check if they understood the text in order to apply as well the method explained. If the first attempt was wrong there is the possibility to update the integrated examples using the randomized variables in Maple T.A. Using this system students can repeat the lecture in their own tempo and even try examples until the grading is correct.

\section{References}

Martin Bicher, Irene Hafner, Andreas Bauer, Carina Pöll, Niki Popper, and Felix Breitenecker. A web-based platform for e-learning and blended learning in modelling and simulation. In International Conference on Business, Technology and Innovation, in Durres, Albanien, S, pages 100-109, 2013.

Andreas Körner, Irene Hafner, Martin Bicher, Stefanie Winkler, and Felix Breitenecker. Mmt - a web environment for education in mathematical modelling and simulation. In Tagungsband Abstracts und Fullpapers, pages 100-109, 2011. ISBN 978-3-905745-44-3.

Maplesoft. Möbius - online courseware environment that puts stem first!, 2016. http://www.maplesoft.com/ products/mobius.

Vilma Urbonaite, Stefanie Winkler, and Andreas Körner. Various usage of maple t.a. in mathematics, modelling and simulation. In ERK - International Electrotechnical and Computer Science Conference, pages 173 - 176, 2013.

Stefanie Winkler, Andreas Körner, and Irene Hafner. Mmt - a web-based elearning system for mathematics, modelling and simulation using Matlab. In Proceedings of the 7th Congress on Modelling and Simulation, pages 1215-1221, 2010. ISBN 978-80-01-04589-3. 